\title{
A METHOD OF CLOSED ORBIT CORRECTION UNDER CONSTRAINT CONDITIONS
}

\author{
K. H KIM, Yujong KIM, C. KIM, J. CHOI, T-Y LEE, \\ Guinyun KIM ${ }^{1}$, M. H. ChO, W. NAMKUnG, and I. S. Ko
}

\author{
Pohang Accelerator Laboratory, POSTECH, Pohang 790-784, Korea \\ ${ }^{1}$ Center of High Energy Physics, Kyungpook National University, Daegu 702-701, Korea
}

\begin{abstract}
We have developed the correction algorithm of the closed orbit distortion (COD) under the constraint condition where beam positions at particular points are not changed. The new algorithm is based on the modified PSINOM algorithm including the regularization process in order to avoid the inversion problem of the ill-posed response matrices. We have confirmed that the COD is well corrected with no orbit leak in the simulation and is in good agreement with the experiment.
\end{abstract}

\section{INTRODUCTION}

When the beam position change takes place during the conventional global orbit correction processes, the photon beam through the beamline is affected, and it results in the alignment of mirrors and monochromators. This is particularly severe for a long beamline such as the undulator beamlines. This problem can be overcome by introducing a local bump at the particular beamline. However, for some light sources, there are not enough corrector magnets to generate local bumps as much as needed. This difficulty can be overcome when we correct the COD under the condition where the beam positions at particular points are not changed. This is our main objective to develop a method of the closed orbit correction under constraint conditions.

\section{THEORY}

\subsection{Ordinary COD correction and regulariza- tion}

The beam position is normally described as a vector $|\mathbf{x}\rangle$ measured by $M$ beam position monitors (BPM). In order to correct the COD, we need $N$ corrector magnets with their strengths described as a vector $|\mathbf{k}\rangle$. When the corrector magnets kick a beam, the new beam positions $|\mathbf{y}\rangle$ can be described as follows.

$$
|\mathbf{y}\rangle=\mathbf{R}|\mathbf{k}\rangle+|\mathbf{x}\rangle .
$$

Here, $\mathbf{R}$ is called the response matrix of $(M \times N)$ dimensions whose components are given by

$$
\mathbf{R}_{i j}=\frac{\sqrt{\beta_{i} \beta_{j}}}{2 \sin \pi \nu} \cos \left(\left|\Psi_{i}-\Psi_{j}\right|-\pi \nu\right),
$$

where $\nu$ is the betatron tune of the storage ring, and $\left(\beta_{i}, \Psi_{i}\right)$ and $\left(\beta_{j}, \Psi_{j}\right)$ are the beta function and the phase function for the $i^{t h}$ BPM and $j^{t h}$ corrector magnet, respectively. In order to reduce the COD, we have to choose the kick of each corrector magnet satisfying

$$
\mathbf{R}^{T} \mathbf{R}|\mathbf{k}\rangle+\mathbf{R}^{T}|\mathbf{x}\rangle=0 .
$$

It is called the PSINOM algorithm[1].

The COD correction is actually a minimization procedure of $S$ defined as

$$
S=\frac{1}{2}\left\{\left\langle\mathbf{k}\left|\mathbf{R}^{T} \mathbf{R}\right| \mathbf{k}\right\rangle+2\langle\mathbf{x}|\mathbf{R}| \mathbf{k}\rangle+\langle\mathbf{x} \mid \mathbf{x}\rangle\right\} .
$$

By using the relations of vector operators which are hyperdimensional gradient operators, we can get the same result of PSINOM algorithm as shown in eq. (3).

This algorithm includes an inversion procedure of the matrix $\mathbf{R}^{T} \mathbf{R}$. In some cases, we can get unacceptable corrections due to the ill-posedness of $\mathbf{R}^{T} \mathbf{R}$. A regularization method is introduced to avoid this problem. In this case, $S$ is written as

$$
\begin{array}{r}
S=\frac{1}{2}\left\{\left\langle\mathbf{k}\left|\mathbf{R}^{T} \mathbf{R}\right| \mathbf{k}\right\rangle+2\langle\mathbf{x}|\mathbf{R}| \mathbf{k}\rangle+\langle\mathbf{x} \mid \mathbf{x}\rangle\right\} \\
+\frac{1}{2}\langle\mathbf{k}|\alpha| \mathbf{k}\rangle .
\end{array}
$$

where $\alpha$ is the regularization parameter. Then the minimum corrector kicks can be determined by

$$
\left(\mathbf{R}^{T} \mathbf{R}+\alpha \mathbf{I}\right)|\mathbf{k}\rangle+\mathbf{R}^{T}|\mathbf{x}\rangle=0 .
$$

This equation represents the modified PSINOM algorithm, and we can relax the inversion problem of the singular matrix by using the diagonal matrix $\alpha \mathbf{I}$ when $\mathbf{R}^{T} \mathbf{R}$ is singular[2].

\subsection{Method with constraint conditions}

After having the new closed orbit with the minimum distortion from eq. (6), the new orbit is generally different from the original orbit. Sometimes, this difference can be taken place at very sensitive locations such as the entrance and the exit of an undulator. If the beamline is well aligned for this undulator, a COD correction should be avoided in this region. A constraint condition can be described in terms of the beam position at $i^{\text {th }}$ BPM such as

$$
\left\langle\mathbf{R}_{i} \mid \mathbf{k}\right\rangle+x_{0 i}=x_{i} .
$$


Here, $\left\langle\mathbf{R}_{i}\right|$ is the $i^{\text {th }}$ row of the response matrix $\mathbf{R}$. Also, $x_{0 i}$ and $x_{i}$ are the beam positions before and after the correction, respectively. Since we want to keep this position unchanged, $\left\langle\mathbf{R}_{i} \mid \mathbf{k}\right\rangle$ should be zero. If there are $L$ BPMs involved in the constraint condition, we can write the constraint condition as follows.

$$
\mathbf{C}^{T}|\mathbf{k}\rangle=0 \text {. }
$$

Here, $\mathbf{C}^{T}$ is the $(L \times N)$ sub-matrix of the response matrix. Each component of $\mathbf{C}^{T}$ corresponds to the BPM involved in the constraint condition. We also assume that $|\mathbf{k}\rangle$ has a non-trivial solution.

We now add this constraint condition to the modified PSINOM algorithm to obtain the new $S$ such as,

$$
\begin{array}{r}
S=\frac{1}{2}\left[\left\langle\mathbf{k}\left|\mathbf{R}^{T} \mathbf{R}\right| \mathbf{k}\right\rangle+2\langle\mathbf{x}|\mathbf{R}| \mathbf{k}\rangle+\langle\mathbf{x} \mid \mathbf{x}\rangle\right] \\
+\frac{1}{2}\langle\mathbf{k}|\alpha| \mathbf{k}\rangle+\left\langle\boldsymbol{\Gamma}\left|\mathbf{C}^{T}\right| \mathbf{k}\right\rangle .
\end{array}
$$

Here, $\langle\boldsymbol{\Gamma}|$ is the Lagrangian multiplier, and it is an $L$ dimensional vector. By following the derivative to the corrector strength, we can get the vector $|\mathbf{k}\rangle$ which minimizes the closed orbit distortion outside the constraint region such as,

$$
(\mathbf{A}+\alpha \mathbf{I})|\mathbf{k}\rangle+\mathbf{R}^{T}|\mathbf{x}\rangle+\mathbf{C}|\mathbf{\Gamma}\rangle=0 .
$$

Here, we define the square matrices of $N \times N$ dimensional A and $L \times L$ dimensional $\mathbf{D}$ as follows.

$$
\begin{array}{r}
\mathbf{A}=\mathbf{R}^{T} \mathbf{R}, \\
\mathbf{D}=\mathbf{C}^{T}(\mathbf{A}+\alpha \mathbf{I})^{-1} \mathbf{C} .
\end{array}
$$

Eq. (10) can be rewritten as

$$
|\boldsymbol{\Gamma}\rangle=-\mathbf{D}^{-1} \mathbf{C}^{T}(\mathbf{A}+\alpha \mathbf{I})^{-1}|\mathbf{x}\rangle .
$$

Now, we can remove the Lagrangian multiplier $|\boldsymbol{\Gamma}\rangle$ in eq. (10) by using the above equation. Then, we can finally get the kick values of the corrector magnets as follows.

$$
\begin{array}{r}
|\mathbf{k}\rangle=-(\mathbf{A}+\alpha \mathbf{I})^{-1}\left\{\mathbf{I}-\mathbf{C D}^{-1} \mathbf{C}^{T}(\mathbf{A}+\alpha \mathbf{I})^{-1}\right\} \\
\mathbf{R}^{T}|\mathbf{x}\rangle .
\end{array}
$$

\section{SIMULATIONS AND EXPERIMENTAL RESULTS}

In order to test the validation of the orbit correction algorithm under the constraint condition, we have conducted the experiment and the computer simulation. The new correction code is written in C language and it is installed in the PLS control system. The code consists of two parts: one for reading BPM signals and setting corrector strengths, and the other for the matrix calculation based on eq. (10). Since the high real-time capability is not necessary in our orbit correction, we execute BPM signal readings and corrector strength settings from the control database in the shared memory of the operator interface

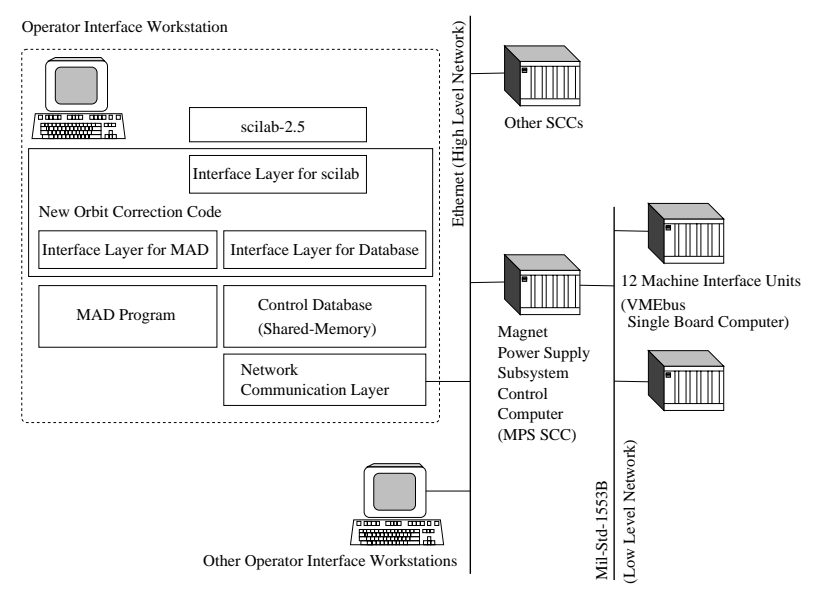

Figure 1: Schematic diagram of the orbit correction code.

workstation. The control database is synchronized the machine control parameter in the real-time VME system via Ethernet. In the matrix calculation, we use the scilab-2.5 code[3]. The configuration for new correction code used in this experiment is illustrated in Fig. 1.

\subsection{Choice of BPMs and correctors}

The PLS lattice consists of 36 bending magnets, 144 quadrupole magnets, 48 sextupole magnets, 72 correctors, and $108 \mathrm{BPMs}$ in the $280-\mathrm{m}$ circumference ring to form a 12-fold symmetry. The response matrix is obtained by reading entire BPM signals while changing the current of a corrector. We select 24 correctors randomly, and we choose a sector containing $40^{\text {th }}$ through $49^{\text {th }}$ BPMs as a constraint region. Finally, 90 BPMs are selected randomly from the remains for this experiment. Ten BPMs in the constraint region are always included in the random selection process.

In order to quantify the result of the orbit correction, we define the rms reduction rate as the ratio of the rms orbit distortion before and after the correction[4]. In this definition, we exclude the orbit distortion in the constraint region because our aim is to preserve the orbit in the constraint region. We also define the rms orbit leak as the rms orbit change before and after the orbit correction in the constraint region. The orbit leak will show how well the constraint is preserved after the orbit correction.

\subsection{Choice of $\alpha$ by simulations}

The next task we have performed is the proper choice of $\alpha$. The simulation is done by using the Methodical Accelerator Design (MAD) code[5] and the scilab-2.5 code[3] for the matrix calculation. We have simulated 30 different orbit distortions for each choice of $\alpha$ when $\alpha$ varies in the range of $0 \leq \alpha \leq 1$, and the results are summarized in Table 1 . When $\alpha$ is too small, we may face a poor inversion, and the rms reduction rate is not affected significantly when $\alpha$ is larger than 0.1 . Therefore, we use the regularization parameter $\alpha$ as 0.01 throughout the experiment and 
Table 1: The RMS orbit reduction and the RMS orbit leak for various $\alpha$ values.

\begin{tabular}{ccc|ccc}
\hline$\alpha$ & reduction & leak $[\mu m]$ & $\alpha$ & reduction & leak $[\mu m]$ \\
\hline \hline 0.0 & 0.513818 & 1.761 & 0.4 & 0.694326 & 1.928 \\
0.001 & 0.513794 & 1.698 & 0.5 & 0.713526 & 1.848 \\
0.01 & 0.504790 & 1.751 & 0.6 & 0.729353 & 1.890 \\
0.05 & 0.548938 & 1.767 & 0.7 & 0.742810 & 1.937 \\
0.1 & 0.586028 & 1.793 & 0.8 & 0.754507 & 1.962 \\
0.2 & 0.636718 & 1.831 & 0.9 & 0.764841 & 2.049 \\
0.3 & 0.669948 & 1.874 & 1.0 & 0.774087 & 1.994 \\
\hline
\end{tabular}

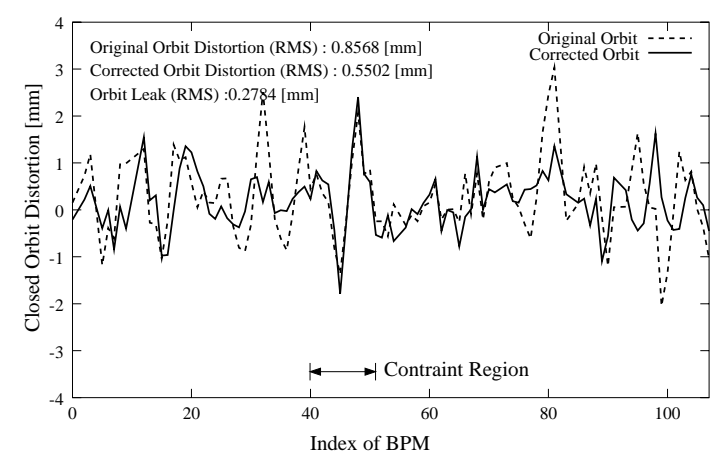

Figure 2: Result of COD correction under the constraint conditions.

the simulation to avoid any singularity.

\subsection{Results}

As a typical example of the orbit correction, Fig. 2 shows that there is little orbit change in the constraint region, while a significant orbit reduction is taken place outside the constraint region. Figure 3 also shows the experimental result of the orbit correction for 30 different orbit distortions. The rms orbit reduction which is the solid line in Fig. 3 is about 0.58 .

This experimental result is compared with the simulation in Fig. 3. In the simulation, we use the same BPMs and correctors for the orbit corrections. The rms orbit reduction in the simulation which is the dotted line in this figure is 0.50 . Although the magnitude of rms orbit distortions in the experiment is larger than the one for the simulation,

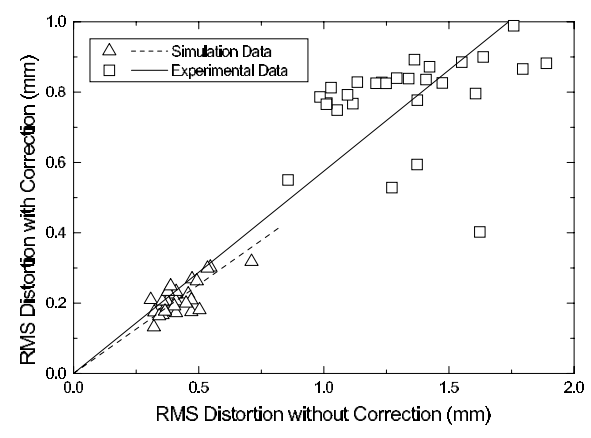

Figure 3: RMS orbit reduction.

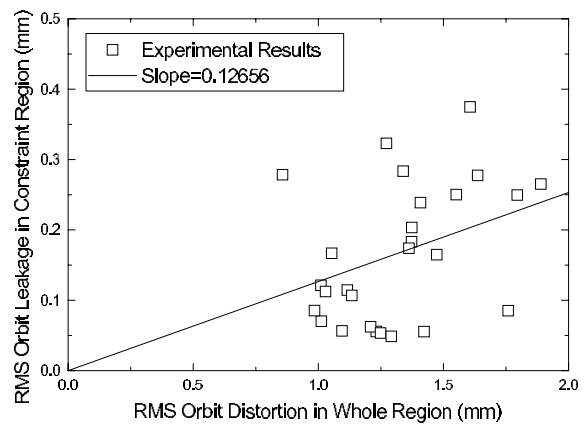

Figure 4: RMS orbit leak.

the experiment is in good agreement with the simulation by counting the nonlinearity and various errors in the real lattice. We also measure the rms orbit leak in the constraint region for 30 different orbit corrections as shown in Fig. 4. It shows the rms orbit leak with respect to the amount of the rms orbit distortion in the entire ring. The leak rate which is the solid line in Fig. 4 is about 0.13 .

\section{CONCLUSION}

We have developed the COD correction algorithm under the constraint condition where the beam position at particular point is not changed. The new algorithm is based on the modified PSINOM algorithm which includes the regularization process in order to avoid the inversion problem of the ill-posed response matrices. There are two parameters introduced during the development of our algorithm. While the Lagrangian multiplier is eliminated by the constraint condition, the parameter $\alpha$ from the regularization process must be determined empirically. We have confirmed that the COD is corrected by about $42 \%$ in the experiment and about $50 \%$ in the simulation. By counting various nonlinearities in real lattice, the COD corrections done by the experiment and by the simulation are in good agreement. Even though the simulation based on the linear lattice gives negligible orbit leaks, it is observed in the experiment that the rms orbit leak is sensitive for the lattice nonlinearity, which needs a further study.

\section{REFERENCES}

[1] W. Herr: "Algorithms and procedures used in the orbit correction package COCU," CERN SL/95-07 (AP), 1995.

[2] Y. N. Tang and S. Krinsky: Proc. AIP Conf. 315 (AIP Press, 1993) 87.

[3] See URL http://www-rocq.inria.fr/scilab.

[4] Kukhee Kim, Kyuyeol Shim, Jinhyuk Choi, Moohyun Cho, Won Namkung and In Soo Ko: Jpn. J. Appl. Phys. 39 (2000) 6094.

[5] F. Christoph Iselin: The MAD Program User's Reference Manual, CERN SL/98 (AP), 1998. 